\title{
Beam quality of partially polarized beams propagating through lenslike birefringent elements
}

\author{
G. Piquero, J. M. Movilla, P. M. Mejías, and R. Martínez-Herrero \\ Departamento de Óptica, Facultad de Ciencias Físicas, Universidad Complutense, 28040 Madrid, Spain
}

Received March 17, 1999; accepted June 29, 1999

\begin{abstract}
Changes generated by lenslike birefringent elements on the beam-quality parameter of partially polarized beams are investigated. Analytical expressions for the beam-quality gain at the output of such systems are given in terms of the second-order intensity moments of the field. Explicit conditions to improve and optimize the beam-quality parameter after propagation through these birefringent transmittances are also shown. (C) 1999 Optical Society of America [S0740-3232(99)00911-4]
\end{abstract}

OCIS codes: $140.0140,140.3280$.

As is well known, the spatial structure of laser beams can be characterized by means of the so-called intensity moments. $^{1-5}$ Within this formalism the possibility of improving the beam-quality parameter under propagation through certain optical elements has been analyzed. Thus, for example, it has been demonstrated that, on the one hand, binary phase plates always degrade the beam quality, ${ }^{6}$ and, on the other hand, quartic phase plates can improve this factor. ${ }^{7}$ But all these previous studies assume a scalar approach (in other words, polarization is disregarded).

In the present paper, however, the vectorial nature of light is taken into account: We investigate the influence that lenslike birefringent (LLB) elements have on the beam-quality parameter. These elements can be regarded (1) as thin lenses made of an anisotropic spatially homogeneous material and (2) as plane-parallel plates made of a birefringent uniaxial medium whose (inhomogeneous) principal indices exhibit a (transversal) quadratic dependence on the distance to the propagation axis. Two applications of such elements include laser resonators designed to obtain predetermined profiles ${ }^{8,9}$ and spatial filtering ${ }^{10}$ (note that such elements can be regarded as pure phase transmittances, thus maintaining the total beam power). After introducing the definitions and the formalism to be used, we give analytical expressions of the beam-quality parameter at the output of LLB elements in terms of the elements' characteristic parameters.

We start with a beam propagating along, say, the $z$ axis, written in terms of its $s$ and $p$ components as follows:

$$
\mathbf{E}(\mathbf{r} ; \mathbf{z})=\left[\mathrm{E}_{\mathrm{s}}(\mathbf{r} ; \mathbf{z}), \mathrm{E}_{\mathrm{p}}(\mathbf{r} ; \mathbf{z})\right],
$$

where $\mathbf{r}=(\mathrm{x}, \mathrm{y})$ denotes the point where the field is evaluated. We define the second-order intensity moments of a partially polarized beam in the form ${ }^{11}$

$$
\begin{aligned}
\langle\alpha \beta\rangle_{\mathrm{j}}= & \frac{1}{\mathrm{P}_{\mathrm{j}}} \iiint \alpha \beta \overline{\mathrm{E}_{\mathrm{j}}^{\dagger}(\mathbf{r}+\mathbf{s} / 2, \mathbf{z}) \mathrm{E}_{\mathrm{j}}(\mathbf{r}-\mathbf{s} / 2, \mathbf{z})} \\
& \times \exp (\mathrm{i} \mathbf{k} \mathbf{s} \boldsymbol{\eta}) \mathrm{d} \mathbf{s d} \mathbf{r d} \boldsymbol{\eta},
\end{aligned}
$$

where the symbol † denotes the adjoint and the overbar represents an ensemble average. In the above equation, $\boldsymbol{\eta}=(\mathrm{u}, \mathrm{v})$ with $\mathrm{u}$ and $\mathrm{v}$ representing angles of propagation (without taking the evanescent waves into account), $\alpha, \beta=\mathrm{x}, \mathrm{y}, \mathrm{u}, \mathrm{v}$, and the subscript $\mathrm{j}$ refers to the $\mathrm{s}$ or $\mathrm{p}$ component. In terms of these measurable moments, the general expression of the beam-quality parameter is given by $^{12}$

$$
\mathrm{Q}_{\mathrm{r}}=\frac{\mathrm{P}_{\mathrm{s}}^{2}}{\mathrm{P}^{2}} \mathrm{Q}_{\mathrm{s}}+\frac{\mathrm{P}_{\mathrm{p}}^{2}}{\mathrm{P}^{2}} \mathrm{Q}_{\mathrm{p}}+\frac{\mathrm{P}_{\mathrm{s}} \mathrm{P}_{\mathrm{p}}}{\mathrm{P}^{2}} \mathrm{Q}_{\mathrm{sp}},
$$

with

$$
\begin{aligned}
\mathrm{Q}_{\mathrm{r}} & =\left\langle\boldsymbol{r}^{2}\right\rangle\left\langle\boldsymbol{\eta}^{2}\right\rangle-\langle\boldsymbol{r} \boldsymbol{\eta}\rangle^{2}, \\
\mathrm{Q}_{\mathrm{s}} & =\left\langle\boldsymbol{r}^{2}\right\rangle_{\mathrm{s}}\left\langle\boldsymbol{\eta}^{2}\right\rangle_{\mathrm{s}}-\langle\boldsymbol{r} \boldsymbol{\eta}\rangle_{\mathrm{s}}^{2}, \\
\mathrm{Q}_{\mathrm{p}} & =\left\langle\boldsymbol{r}^{2}\right\rangle_{\mathrm{p}}\left\langle\boldsymbol{\eta}^{2}\right\rangle_{\mathrm{p}}-\langle\mathbf{r} \boldsymbol{\eta}\rangle_{\mathrm{p}}^{2}, \\
\mathrm{Q}_{\mathrm{sp}} & =\left\langle\boldsymbol{r}^{2}\right\rangle_{\mathrm{s}}\left\langle\boldsymbol{\eta}^{2}\right\rangle_{\mathrm{p}}+\left\langle\mathbf{r}^{2}\right\rangle_{\mathrm{p}}\left\langle\boldsymbol{\eta}^{2}\right\rangle_{\mathrm{s}}-2\langle\boldsymbol{r} \boldsymbol{\eta}\rangle_{\mathrm{s}}^{2}\langle\mathbf{r} \boldsymbol{\eta}\rangle_{\mathrm{p}}^{2}
\end{aligned}
$$

where $P_{s}$ and $P_{p}$ are the radiant powers associated with the $s$ and $p$ components, respectively, and $P=P_{s}+P_{p}$. As usual, $\left\langle\mathbf{r}^{2}\right\rangle,\left\langle\boldsymbol{\eta}^{2}\right\rangle$ and $\langle\mathbf{r} \boldsymbol{\eta}\rangle$ are connected to the beam width, the far-field divergence, and the inverse of the effective radius of curvature of each field component. Note that $Q_{r}$ is related to the standard beam-propagation factor $\mathrm{M}^{2}$ through the expression $\left(\mathrm{M}^{2}\right)^{2}=\mathrm{k}^{2} \mathrm{Q}_{\mathrm{r}}$.

Within the framework of the $\mathrm{J}$ ones matrix treatment, to determine the field amplitude at the output plane of the birefringent plate we will make use of the paraxial approximation (which is consistent with the intensitymoment treatment), and assume $t / z_{R} \ll 1$, where $t$ is the maximum thickness of the optical element along the $z$ direction and $z_{R}$ is the Rayleigh range of the beam. In such a case, the beam width $w(z)$ can be assumed to re- 
main constant inside the plate. In the calculations we consider that the orientation of the optic axis of the anisotropic (uniaxial) medium is constant and perpendicular to the propagation direction of the beam ( $z$ axis). For the sake of simplicity (but without loss of generality) we handle a reference coordinate system in which the $s$ axis coincides with the optic axis and accordingly, the $p$ axis is perpendicular to the $s$ and $z$ directions. Furthermore, the incident beam is assumed to be centered at the $z$ axis; that is, $\langle\mathrm{x}\rangle_{\mathrm{s}, \mathrm{p}}=\langle\mathrm{y}\rangle_{\mathrm{s}, \mathrm{p}}=\langle\mathrm{u}\rangle_{\mathrm{s}, \mathrm{p}}=\langle\mathrm{v}\rangle_{\mathrm{s}, \mathrm{p}}=0$.

Taking the above into account, the field amplitude at the output of a LLB element (modeled as a thin, pure phase transmittance) takes the form

$$
\begin{aligned}
& E_{s}^{(o)}(x, y)=\exp \left[i k \phi_{s}(x, y)\right] E_{s}^{(i)}(x, y), \\
& E_{p}^{(o)}(x, y)=\exp \left[i k \phi_{p}(x, y)\right] E_{p}^{(i)}(x, y),
\end{aligned}
$$

where the superscripts (o) and (i) refer to the output and the input beam, respectively, and

$$
\begin{aligned}
& \phi_{\mathrm{s}}(\mathrm{x}, \mathrm{y})=\mathrm{c}\left(\mathrm{x}^{2}+\mathrm{y}^{2}\right), \\
& \phi_{\mathrm{p}}(\mathrm{x}, \mathrm{y})=\mathrm{d}\left(\mathrm{x}^{2}+\mathrm{y}^{2}\right),
\end{aligned}
$$

$c$ and $d$ being constants, proportional to the extraordinary and the ordinary refractive index of the medium $\left(\mathrm{n}_{\mathrm{e}}\right.$ and $\mathrm{n}_{\mathrm{o}}$ ), respectively. In the particular case in which the LLB element is an anisotropic planoconvex lens, we have $d-c=\left(n_{0}-n_{e}\right) / R_{L}$, where $R_{L}$ represents the curvature radius of the lens.

From Eqs. (1)-(7) it can be shown after lengthy but straightforward calculations that the beam-quality parameter $Q_{r}$ at the output plane of the birefringent system is given by

$$
Q_{r}^{(0)}=Q_{r}^{(i)}+\frac{4 P_{s} P_{p}}{P^{2}}(K+M),
$$

where

$$
\begin{aligned}
& \mathrm{K}=(\mathrm{d}-\mathrm{c})^{2}\left\langle\mathbf{r}^{2}\right\rangle_{\mathrm{s}}^{(\mathrm{i})}\left\langle\mathbf{r}^{2}\right\rangle_{\mathrm{p}}^{(\mathrm{i})}, \\
& \mathrm{M}=(\mathrm{d}-\mathrm{c})\left[\left\langle\mathbf{r}^{2}\right\rangle_{\mathrm{s}}^{(\mathrm{i})}\langle\mathbf{r} \boldsymbol{\eta}\rangle_{\mathrm{p}}^{(\mathrm{i})}-\left\langle\mathbf{r}^{2}\right\rangle_{\mathrm{p}}^{(\mathrm{i})}\langle\mathbf{r} \boldsymbol{\eta}\rangle_{\mathrm{s}}^{(\mathrm{i})}\right] .
\end{aligned}
$$

A number of consequences can be inferred from Eq. (12):

1. The case $(\mathrm{d}-\mathrm{c})=0$ corresponds to an isotropic quadratic phase transmittance. The beam-quality parameter remains invariant, as expected. Note that this result coincides with that obtained in the scalar case.

2. When $(d-c) \neq 0$ but $M=0$, the beam quality always degrades because $K>0$. The beam quality gain $\left(\Delta \mathrm{Q}_{\mathrm{r}}\right)$ then becomes

$$
\Delta Q_{r}=Q_{r}^{(0)}-Q_{r}^{(i)}=\frac{4 P_{s} P_{p}}{P^{2}}(d-c)^{2}\left\langle r^{2}\right\rangle_{s}^{(i)}\left\langle r^{2}\right\rangle_{p}^{(i)} .
$$

The condition $\mathrm{M}=0$ is fulfilled provided that the curvature radii ${ }^{13}$ of the beam associated with the $s$ and $p$ components are the same at the anisotropic plate; i.e., $R_{s}^{(i)}$ $=\langle\mathbf{r} \boldsymbol{\eta}\rangle_{\mathrm{s}}^{(\mathrm{i})} /\left\langle\mathbf{r}^{2}\right\rangle_{\mathrm{s}}^{(\mathrm{i})}=\langle\mathbf{r} \boldsymbol{\eta}\rangle_{\mathrm{p}}^{(\mathrm{i})} /\left\langle\mathbf{r}^{2}\right\rangle_{\mathrm{p}}^{(\mathrm{i})}=\mathrm{R}_{\mathrm{p}}^{(\mathrm{i})}$. Uniformly totally polarized beams and nonpolarized beams are examples of this kind of field.

In the particular case of linearly polarized beams propagating through a birefringent lens, we get

$$
\Delta \mathrm{Q}_{\mathrm{r}}=4 \cos ^{2} \alpha \sin ^{2} \alpha\left(\frac{\mathrm{n}_{\mathrm{o}}-\mathrm{n}_{\mathrm{e}}}{\mathrm{R}_{\mathrm{L}}}\right)^{2}\left[\left\langle\mathbf{r}^{2}\right\rangle^{(\mathrm{i})}\right]^{2},
$$

where $\alpha$ denotes the azimuth of the polarization plane with respect to the s axis. To write Eq. (16) we have used the fact that $\left\langle\mathbf{r}^{2}\right\rangle_{\mathrm{s}}^{(\mathrm{i})}=\left\langle\mathbf{r}^{2}\right\rangle_{\mathrm{p}}^{(\mathrm{i})}=\left\langle\mathbf{r}^{2}\right\rangle^{(\mathrm{i})}$ for uniformly totally polarized beams.

3. Note that both $\mathrm{K}$ and $\mathrm{M}$ depend on $\mathrm{z}$; that is, they depend on the plane where we place the LLB element. Since $K>0$, the beam quality will then degrade for any $z$ if $M(z)=$ constant. To see this, note first that

$$
\begin{aligned}
M(z)= & M(0)+z(d-c)\left[\left\langle\mathbf{r}^{2}\right\rangle_{s}^{(i)}\left\langle\boldsymbol{\eta}^{2}\right\rangle_{p}^{(i)}-\left\langle\mathbf{r}^{2}\right\rangle_{p}^{(i)}\left\langle\boldsymbol{\eta}^{2}\right\rangle_{s}^{(i)}\right] \\
& +z^{2}(d-c)\left[\langle\mathbf{r} \boldsymbol{\eta}\rangle_{s}^{(i)}\left\langle\boldsymbol{\eta}^{2}\right\rangle_{p}^{(i)}-\left\langle\mathbf{r}^{2}\right\rangle_{p}^{(i)}\left\langle\boldsymbol{\eta}^{2}\right\rangle_{s}^{(i)}\right],
\end{aligned}
$$

where we are assuming free propagation from the plane $\mathrm{z}=0$. Consequently, the condition $\mathrm{M}(\mathrm{z})=$ constant implies that

$$
\begin{gathered}
\left\langle\mathbf{r}^{2}\right\rangle_{\mathrm{s}}^{(\mathrm{i})}\left\langle\boldsymbol{\eta}^{2}\right\rangle_{\mathrm{p}}^{(\mathrm{i})}=\left\langle\mathbf{r}^{2}\right\rangle_{\mathrm{p}}^{(\mathrm{i})}\left\langle\boldsymbol{\eta}^{2}\right\rangle_{\mathrm{s}}^{(\mathrm{i})}, \\
\langle\mathbf{r} \boldsymbol{\eta}\rangle_{\mathrm{s}}^{(\mathrm{i})}\left\langle\boldsymbol{\eta}^{2}\right\rangle_{\mathrm{p}}^{(\mathrm{i})}-\langle\mathbf{r} \boldsymbol{\eta}\rangle_{\mathrm{p}}^{(\mathrm{i})}\left\langle\boldsymbol{\eta}^{2}\right\rangle_{\mathrm{s}}^{(\mathrm{i})}=0 .
\end{gathered}
$$

It then follows directly

$$
M(0)=0,
$$

which, in turn, implies that

$$
M(z)=0 .
$$

In particular, conditions (18) and (19) are fulfilled by both uniformly totally polarized beams and nonpolarized fields. Also note that such conditions are invariant under rotation about the $z$ axis.

As a consequence of the above, for those beams whose intensity moments do not satisfy Eqs. (18) and (19), it could be possible to improve their beam quality by placing the LLB element at an appropriate plane [provided that $M(z)$ differs from zero).

4. When $(d-c) \neq 0, M \neq 0$, and $K+M<0$, the beam-quality parameter can be improved. This condition is fulfilled whenever

$$
|(\mathrm{d}-\mathrm{c})|=\left|\frac{\left(\mathrm{n}_{\mathrm{o}}-\mathrm{n}_{\mathrm{e}}\right.}{\mathrm{R}_{\mathrm{L}}}\right|<\left|\frac{1}{\mathrm{R}_{\mathrm{s}}^{(\mathrm{i})}}-\frac{1}{\mathrm{R}_{\mathrm{p}}^{(\mathrm{i})}}\right|,
$$

where the second member of this equation applies to planoconvex uniaxial lenses.

5. The optimum value of the beam-quality parameter is attained when

$$
2(\mathrm{~d}-\mathrm{c})=\frac{1}{\mathrm{R}_{\mathrm{s}}^{(\mathrm{i})}}-\frac{1}{\mathrm{R}_{\mathrm{p}}^{(\mathrm{i})}} .
$$

In this case, the beam-quality gain takes the form

$$
\left(\Delta Q_{r}\right)_{\text {opt }}=-\frac{\mathrm{P}_{\mathrm{s}} \mathrm{P}_{\mathrm{p}}}{\mathrm{P}^{2}}\left\langle\mathbf{r}^{2}\right\rangle_{\mathrm{s}}^{(\mathrm{i})}\left\langle\mathbf{r}^{2}\right\rangle_{\mathrm{p}}^{(\mathrm{i})}\left[\frac{1}{\mathrm{R}_{\mathrm{s}}^{(\mathrm{i})}}-\frac{1}{\mathrm{R}_{\mathrm{p}}^{(\mathrm{i})}}\right]^{2},
$$

which depends on the values of the second-order moments at the plane where the LLB element is placed.

Finally, we remark that the above expressions are not invariant under rotation of the LLB element about the propagation axis. Accordingly, improvement in the qual- 
ity is rotationally dependent. Once the beam moments at the input plane are known (either theoretically or experimentally) for a certain coordinate system, their values can be determined for any other orientation $\theta$ of the coordinate axes after application of the corresponding rotation matrix. One can then infer the optimal LLB element and the best orientation of its optic axis from the values of $\mathrm{R}_{\mathrm{s}}^{(\mathrm{i})}$ and $\mathrm{R}_{\mathrm{p}}^{(\mathrm{i})}$ for any $\theta$ by using Eq. (24).

\section{ACKNOWLEDGMENTS}

This work was supported by the Comisión Interministerial de Ciencia y Tecnología of Spain under project PB970295.

G. Piquero can be reached at the address on the title page or by phone, 34-91-394-4678; fax, 34-91-394-4683; or e-mail, piquero@eucmax.sim.ucm.es.

\section{REFERENCES}

1. S. Lavi, R. Prochaska, and E. Keren, “Generalized beam parameters and transformation law for partially coherent light," Appl. Opt. 27, 3696-3703 (1988).

2. M. J. Bastiaans, "Propagation laws for the second-order moments of the Wigner distribution function in first-order optical systems," Optik (Stuttgart) 82, 173-181 (1989).

3. A. E. Siegman, "N ew developments in laser resonators," in
Optical Resonators, D. A. Holmes, ed., Proc. SPIE 1224 2-14 (1990).

4. J. Serna, R. Martínez-Herrero, and P. M. Mejías, "Parametric characterization of general partially coherent beams propagating through ABCD optical systems," J . Opt. Soc. Am. A 8, 1094-1098 (1991).

5. H. Weber, "Propagation of higher-order intensity moments in quadratic-index media," Opt. Quantum Electron. 24, 1027-1049 (1992)

6. A. E. Siegman, "Binary phase plates cannot improve laser beam quality," Opt. Lett. 18, 675-677 (1993).

7. R. Martínez-Herrero, P. M. Mejías, and G. Piquero, "Quality improvement of partially coherent symmetric-intensity beams caused by quartic phase distortions," Opt. Lett. 17, 1650-1651 (1992).

8. B. J . Feldman and S. J . Gitomer, "Anular lens soft aperture for high power laser systems," Appl. Opt. 15, 1379-1380 (1976).

9. G. Giuliani, Y. K. Park, and R. L. Byer, "Radial birefringent element and its application to laser resonator design," Opt. Lett. 5, 491-493 (1980).

10. J . M. Eggleston, G. Giuliani, and R. L. Byer, "Radial intensity filters using radial birefringent elements,"J . Opt. Soc. Am. 71, 1264-1272 (1981).

11. R. Martínez-Herrero, P. M. Mejías, and J . M. Movilla, "Spatial characterization of general partially polarized beams," Opt. Lett. 22, 206-208 (1997).

12. Q. Lü, S. Dong, and H. Weber, "Analysis of TEM $M_{00}$ laser beam degradation caused by a birefringent Nd:YAG rod," Opt. Quantum Electron. 27, 777-783 (1995).

13. P. A. Bélanger, "Beam propagation and the $A B C D$ ray matrices," Opt. Lett. 16, 196-198 (1991). 